\title{
ESTUDO DO PERFIL DE DISTRIBUIÇÃO DE TAMANHO E NÚMERO DE PARTÍCULAS EMITIDAS NA QUEIMA DE MISTURAS DE DIESEL/BIODIESEL/ETANOL
}

\author{
Lílian Lefol Nani Guarieiro ${ }^{1,5^{*}}$, Egídio Teixeira de Almeida Guerreiro ${ }^{1,5}$, Keize Katiane dos \\ Santos Amparo', Danilo J. Leão ${ }^{2}$, Ednildo A. Torres ${ }^{3,4,5}$, Jailson B. de Andrade ${ }^{2,4,5}$ \\ ${ }^{1}$ SENAI CIMATEC , 41650-010, Salvador-BA, Brasil; \\ ${ }^{2}$ Universidade Federal da Bahia, Instituto de Química, 40170290 Salvador, BA, Brasil; \\ ${ }^{3}$ Universidade Federal da Bahia, Escola Politécnica, 40210-730 Salvador, BA, Brasil; \\ ${ }^{4}$ Centro Interdisciplinar de Energia e Ambiente (CIEnAm), 40110-040 Salvador, BA, Brasil; \\ ${ }^{5}$ INCT de Energia e Ambiente, UFBA, 40170-290 Salvador, BA, Brasil \\ E-mails: *lilianguarieiro@gmail.com
}

\section{RESUMO}

Neste trabalho são apresentados resultados do estudo do uso de um contador de partículas modelo 3910 TSI na determinação da distribuição de número e tamanho de partículas (tamanho de $11,5 \mathrm{~nm}$ a $365,2 \mathrm{~nm}$ ) emitidas na exaustão de motores diesel utilizando combustíveis com diferentes teores de oxigênio (B5, B5 com 6\% de etanol e B100). A média geométrica para ambos os combustíveis foi $\sigma=86,6+3,7 \mathrm{~nm}$, com número total de partículas (partículas/cm3) 9,6 x 106 (B5), 1,1 x 107 (B5 com 6\% de etanol) e 1,4 x 107 (B100). Uma análise de componentes principais foi aplicada na matriz de dados obtida, 10 x 75. A análise de PCA indica que o grupo com valores de escores maiores e negativos (combustível B5) apresentou maior emissão de partículas de diâmetro maiores. O aumento do teor de oxigênio do combustível destinado a motores ciclo diesel pode influenciar na redução do tamanho das partículas emitidas em sua queima e no aumento da concentração destas, a depender do tipo de câmara de combustão do motor.

\section{INTRODUÇÃO}

Os motores que utilizam diesel como combustível encontram bastantes aplicações, devido principalmente à sua maior eficiência térmica e economia de combustível. No entanto a emissão de poluentes desses motores se tornou uma das maiores preocupações devido ao seu impacto ambiental [1,2]. Nos últimos anos a legislação que regulamenta a emissão dos poluentes tem obrigado fabricantes e montadoras a desenvolverem motores e automóveis menos poluentes. Nesse cenário os combustíveis provenientes de fontes renováveis ganharam bastante destaque e surgiram como possíveis alternativas ao uso de combustíveis fósseis. Vários testes já foram realizados com o biodiesel a fim de conhecer os impactos causados no desempenho dos motores, consumo de combustível e principalmente na emissão de poluentes em relação ao diesel [2,3].

As diferenças nas características do desempenho, combustão e emissão do biodiesel são causadas pela diferença das propriedades químicas e termofísicas existentes entre este e o 
diesel proveniente do petróleo, como a densidade, número de cetanas e teor de oxigênio, maiores no biodiesel que no diesel [1,2].

Apesar de não existir correlação linear entre a utilização de maiores teores de biodiesel em motores diesel, em algumas pesquisas pode-se observar que o uso de biodiesel melhora o processo de combustão e reduz as emissões de hidrocarbonetos (HC) [2]. Outras possíveis explicações para a diminuição de hidrocarbonetos não queimados é devido ao maior teor de oxigênio na molécula de biodiesel, que leva à uma combustão mais limpa e completa [2-4]. $\mathrm{O}$ maior número de cetano do biodiesel reduz o avanço da combustão resultando assim na queda das emissões de HC.

As partículas oriundas da combustão de motores de combustão interna (Material Particulado MP) são constituídas de grandes aglomerados carbonáceos sólidos, compostos de enxofre e compostos orgânicos voláteis. O MP é o principal agente na causa e no agravamento de doenças crônicas do aparelho respiratório dos seres humanos, tal toxicidade depende da composição dessas partículas e principalmente do seu diâmetro aerodinâmico. As partículas consideradas ultrafinas, ou seja, quando o seu diâmetro é inferior ou igual a $100 \mathrm{~nm}$, as quais são capazes de ultrapassar as barreias do aparelho respiratório dos seres humanos e alcançar a corrente sanguínea, são consideradas as mais nocivas dentre todas as outras faixas de tamanho emitidas por motores de combustão interna $[5,6]$.

Nesse sentido, o presente trabalho tem como objetivo o levantamento do perfil da distribuição de numero e tamanho das partículas emitida por um motor ciclo diesel, utilizando diferentes misturas de combustíveis contendo variados teores de oxigênio verificando a influencia desse teor na emissão de material particulado.

\section{MATERIAS E MÉTODOS}

No estudo da distribuição de tamanho e numero de partículas foi utilizado um motor de combustão interna ciclo diesel AGRALE, e suas características estão descritas na Tabela 1.

Tabela 1 - Principais especificações do motor.

\begin{tabular}{ll}
\hline Especificações & N790 \\
\hline Modelo & 2 \\
$\mathrm{~N}^{\circ}$ de cilindros & Agrale \\
Fabricante & $1272 \mathrm{~cm}^{3}$ \\
Volume & $20: 1$ \\
Taxa de compressão & $63,02 \mathrm{Nm}$ \\
Torque & Direta mecânica \\
Sistema de injeção & $19,8 \mathrm{Kw}$ a 3000rpm \\
Potência & A ar \\
Refrigeração &
\end{tabular}

O motor foi acoplado a um dinamômetro hidráulico da marca SCHENK para aplicação de carga por meio de um eixo do tipo cardã (Figura 1). As condições de operação do motor 
durante os testes foram estabelecidas em uma rotação fixa de $1800 \mathrm{rpm}$ e uma carga de $70 \%$. Variáveis como temperatura ambiente, temperatura dos gases do escapamento, bem como a umidade do ar ambiente também foram medidas por sensores apropriados.

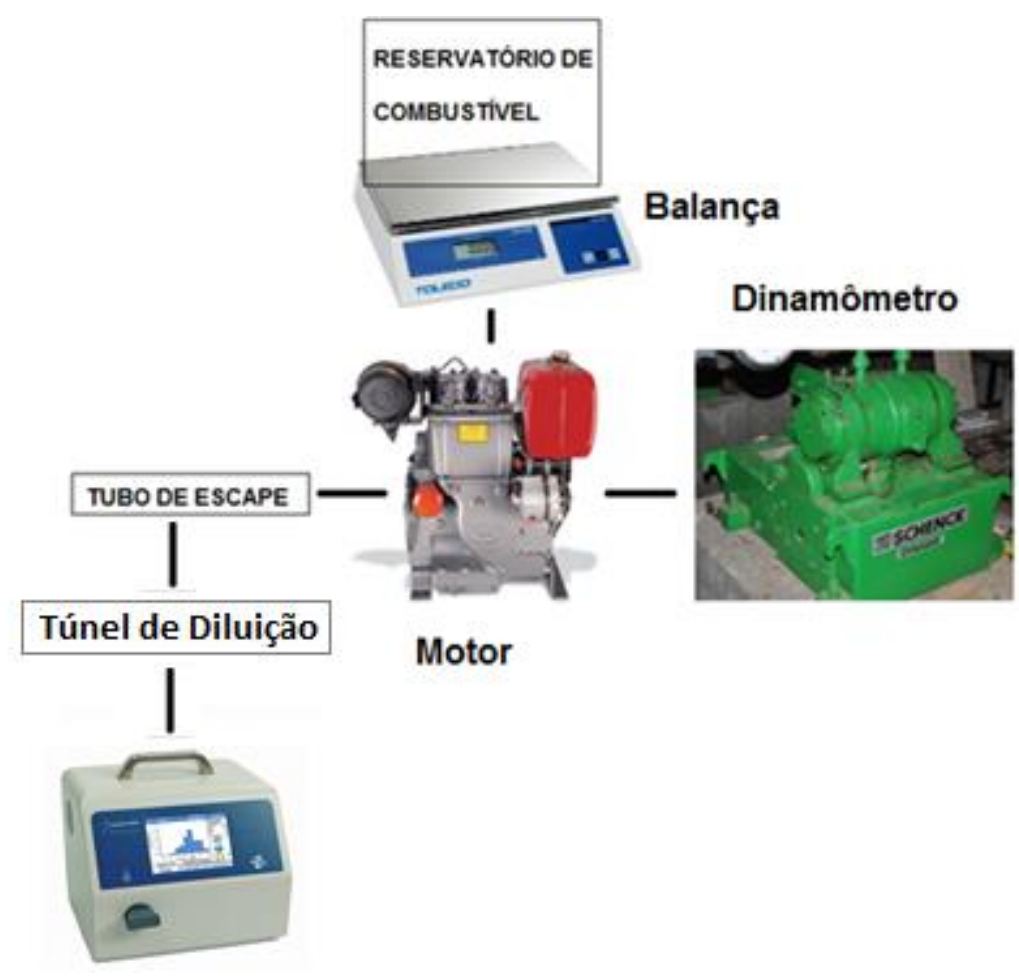

Figura 1 - Diagrama da bancada de testes

Para a coleta de amostras de material particulado foi acoplado ao escapamento do motor um túnel de diluição parcial a volume constante, com taxa de diluição de, onde foi conectado um contador de partículas modelo 3910 da fabricante TSI, avaliando partículas na faixa de diâmetros entre $11,5 \mathrm{~nm}$ e $365,2 \mathrm{~nm}$.

O biodiesel de soja utilizado para compor as misturas foi doado pela Petrobahia. (Distribuidora de Petróleo da Bahia S.A.). O álcool etílico pureza 99,3\% foi fornecido pela Pro-Análise ${ }^{\circledR}$ e a mistura binária (B5) foi obtida localmente em postos de abastecimento da Petrobrás®. A Tabela 2 apresenta as especificações dos combustíveis utilizados.

Nos estudo da distribuição de tamanho e número de partículas foram utilizados três diferentes tipos de combustíveis: biodiesel puro (B100), diesel com 5\% de biodiesel (B5) e uma mistura ternária contendo $89 \%$ de diesel, 5\% de biodiesel e 6\% de etanol (B5E6). A mistura ternária B5E6 foi preparada em laboratório.

Tabela 2 - Especificação dos combustíveis utilizados.

\begin{tabular}{lcccc}
\hline Characteristics & B5 & B5E6 & B100 & Ethanol \\
\hline Densidade $\left(\mathrm{g} \mathrm{mL}^{-1}, 20^{\circ} \mathrm{C}\right)$ & 0.853 & 0.821 & 0.870 & 0.790 \\
\hline
\end{tabular}




\begin{tabular}{lcccc}
\hline Viscosidade $\left(\mathrm{cSt}, \mathrm{at}{ }^{40} \mathrm{C}\right)$ & 4.12 & 3.40 & 4.20 & 1.18 \\
Calor latent de vaporização $\left({\left.\mathrm{kJ} . \mathrm{kg}^{-1}\right)}\right.$ & 270 & 350 & 200 & 840 \\
Número de Cetano & 48 & 41 & 56 & 6 \\
Poder Calorífico Inferior (kJ.kg-1) & 42820 & 41733 & 36395 & 28300 \\
\hline
\end{tabular}

Os testes no motor seguiram um procedimento padrão para os ensaios dos três combustíveis. Primeiramente o motor foi estabilizado por cerca de 30 minutos com o combustível a ser ensaiado. Após esse período foram fixadas as condições de teste no motor (1800rpm e carga de $70 \%)$.

Para a coleta do material particulado foi realizado previamente uma amostragem do túnel de diluição ainda com o motor desligado, a fim de identificar as partículas existentes no ar de diluição, rastreando assim suas possíveis influencias durantes as amostragens com os combustíveis. Foram coletadas 10 amostras em triplicata, com duração de 10 min cada e intervalos de 15 min entre elas. Filtros presente nos sistemas de aquisição e controle foram trocados a cada novo inicio de coleta com diferentes combustíveis.

\section{RESULTADOS E DISCURSÕES}

A queima dos combustíveis utilizados durante as coletas apresentou concentrações de partículas na moda de acumulação de $50<\mathrm{Da}<200 \mathrm{~nm}$ (Figura 2). A média geométrica para os combustíveis B5 e B5E6 foi de $\delta=86,6 \pm 3,7 \mathrm{~nm}$ com um numero total de partículas de $9,6 \times 10^{6}$ partículas $/ \mathrm{cm}^{3}$ para o B5 e $1,1 \times 10^{7}$ partículas $/ \mathrm{cm}^{3}$ para o B5E6. A média geométrica obtida para o combustível B100 foi de $\delta=78.1 \pm 3.1 \mathrm{~nm}$ com um número total de partículas de $1,4 \times 10^{7}$ partículas $/ \mathrm{cm}^{3}$.

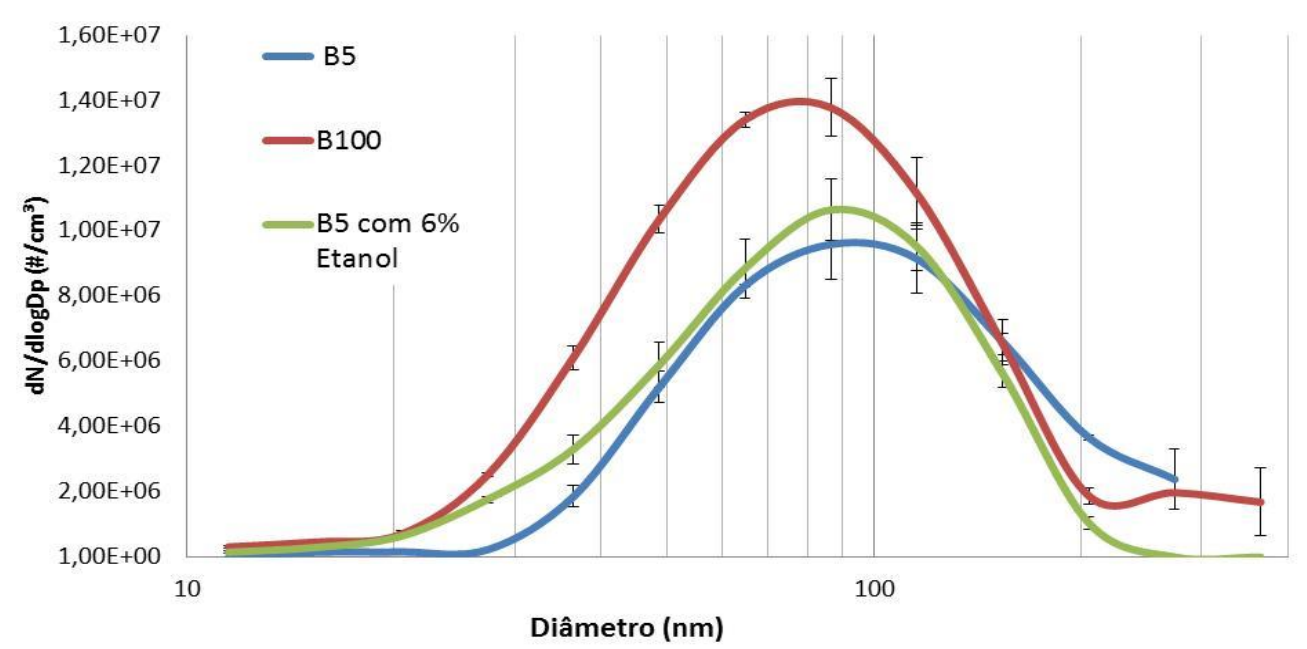

Figura 2 - Distribuição de número e tamanho de partículas para os três combustíveis estudados. 
Através dos resultados obtidos pode-se concluir que o teor de oxigênio presente no combustível pode estar ocasionando um deslocamento da mediana refletindo no aumento da emissão de número de partículas menores e redução da emissão do número de partículas maiores (Figura 2).

Aplicando uma analise de componentes principais na matriz de dados das amostras, foi encontrado que a variância acumulada por PC1 e PC2 foi de 84,21\% com formação de três grupos de distribuição (Figura 3 e 4).

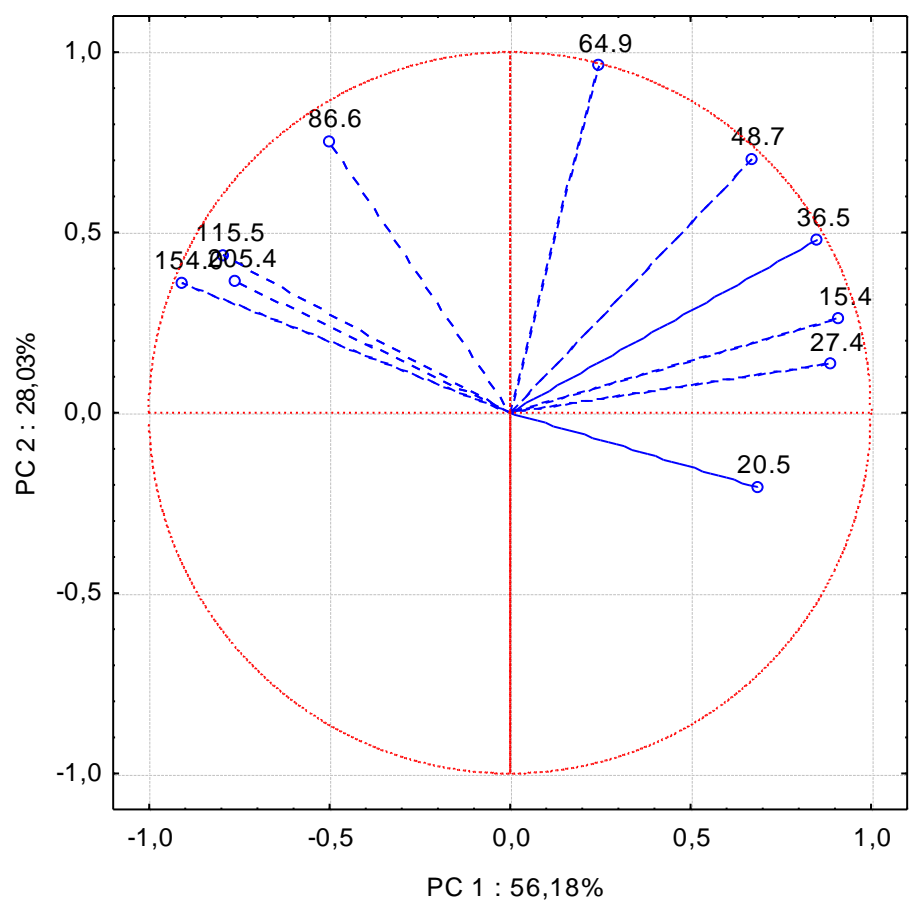

Figura 3 - Gráfico de pesos das misturas de combustíveis.

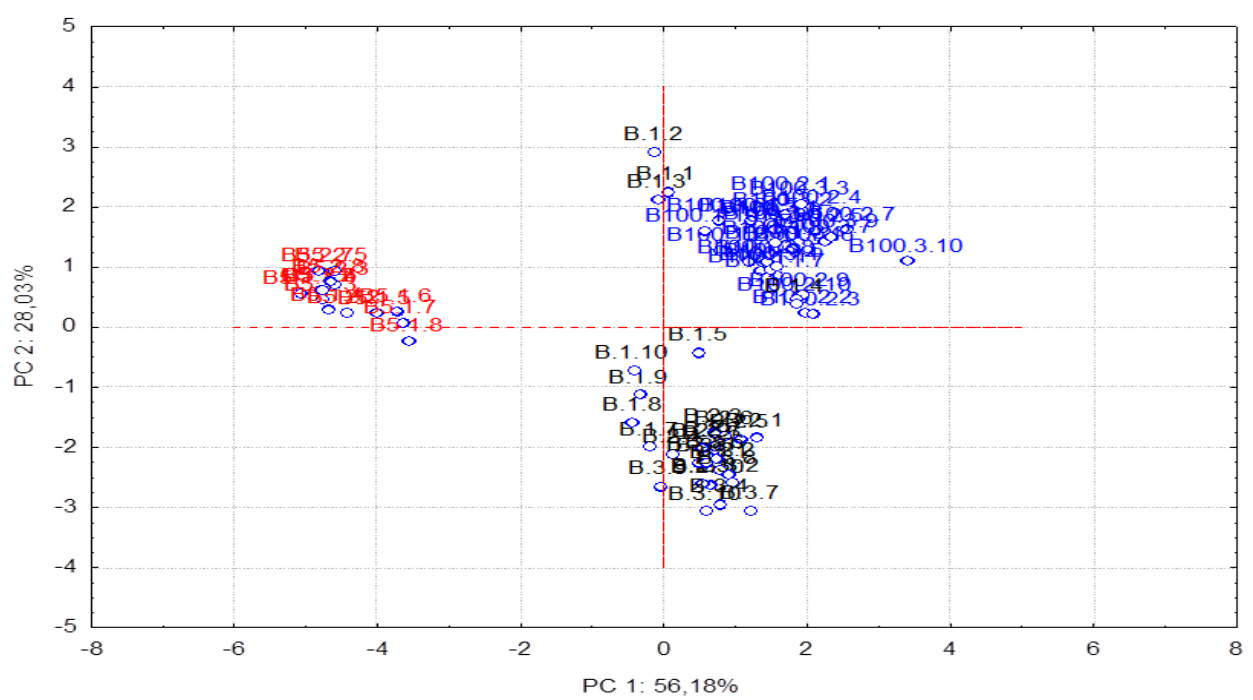

Figura 4 - Resultados de escores das misturas de combustíveis. 
Como resultado da analise de PCA é possível identificar que o grupo com valores de escores maiores e negativos (B5) apresentou uma maior emissão de partículas de diâmetro maiores enquanto que os outros combustíveis B100 e B5E6 (deslocados mais para a direita do gráfico) apresentaram uma maior emissão de partículas com diâmetros menores (Figura 4). Os resultados obtidos neste trabalho estão de acordo com os resultados obtidos por Tsolakis [7] e Cheng [8], onde foram observados também aumento na emissão de número de partículas menores quando se compara a queima de biodiesel com a queima de diesel e mistura diesel/etanol.

Além do teor de oxigênio maior do B100 em relação aos combustíveis estudados neste trabalho, o motor utilizado trabalho possui injeção mecânica. Assim, existe uma injeção antecipada por conta do alto modulo de compressibilidade volumétrica do biodiesel e isso faz com que o biodiesel tenha mais tempo para se misturar com o ar, produzindo uma queima mais completa e, consequentemente, reduzindo o tamanho das partículas emitidas e aumentando sua concentração.

\section{CONCLUSÃO}

O aumento do teor de oxigênio do combustível destinado a motores ciclo diesel pode influenciar na redução do tamanho das partículas emitidas em sua queima e no aumento da concentração destas. Além disso, o tipo de câmara de combustão do motor também pode influenciar na redução do tamanho das partículas emitidas.

\section{AGRADECIMENTOS}

Os autores agradecem ao Instituto Nacional de Ciência e Tecnologia de Energia e Ambiente (INCT EA); Projeto Bahia de Todos os Santos (Portal Kirimurê), ao Conselho Nacional de Desenvolvimento Científico e Tecnológico $(\mathrm{CNPq})$ e a Fundação de Amparo à Pesquisa do Estado da Bahia (FAPESB).

\section{REFERÊNCIAS}

[1] AN, H; YANG, W. M; CHOU, S, K; CHUA, K, J. Combustion and emissions characteristics of diesel engine fueled by biodiesel at partial load conditions, Applied Energy, v.99, p.363-371, 2012.

[2] GUARIEIRO, L. L. N.; GUARIEIRO, A. L. N. Vehicle Emissions: What Will Change with Use of Biofuel? INTECH, Biofuels - Economy, Environment and Sustainability, Chapter 14, p. 357-386, 2012.

[3] IMTENAN, S.; MASJUKI, H.H.; VARMAN, M.; KALAM, M.A.; ARBAB, M.I.; SAJJAD, H.; ASHRAFUR RAHMAN, S.M. Impact of oxygenated additives to palm and jatropha biodiesel blends in the context of performance and emissions characteristics of a light-duty diesel engine, Energy Conversion and Management, v. 83, p. 149-158, 2014. 
[4] LAPUERTA, M., ARMAS, O., FERNANDEZ, J. R.. "Effect of biodiesel fuels on diesel engine emissions, Progress in energy and combustion science v. 34, p. 198-223, 2008.

[5] GUARIEIRO, L.L.N.; VASCONCELlOS, P.C.; Solci, M.C.. Poluentes Atmosféricos Provenientes da Queima de Combustíveis Fósseis e Biocombustíveis: Uma Breve Revisão. Revista Virtual de Química. v. 3(5), p. 434-445, 2011.

[6] D. MARTINS, ; C. R. S. JÚNIOR, M. C. SOLCI, J. P. PINTO, D. Z. SOUZA, P. VASCONCELLOS, A. L. N. GUARIEIRO, L. L. N. GUARIEIRO, E. T. SOUSA, J. B. DE ANDRADE, Particle emission from heavy-duty engine fuelled with blended diesel and biodiesel, Environ. Monit. Assess. v. 184, p. 2663-2676, 2012.

[7] TSOLAKIS, A. Effects on particle size distribution from the diesel engine operating on RME-biodiesel with EGR, Energ Fuel, v. 20, p. 1418-1424, 2006.

[8] CHENG, C.S. Comparison of emissions of a direct injection diesel Engine operating on biodiesel with emulsified and fumigated metanol, Fuel, v. 87, p. 1870-1879, 2008. 